\title{
Deutschsprachiger AOTrauma Master Kurs 2016
}

\author{
Paul Alfred Grützner
}

Von Sonntag, 4. Dezember bis Donnerstag, 8. Dezember 2016 findet in Davos der einzige deutschsprachige AOTrauma Master Kurs weltweit statt.

Der deutschsprachige AOTrauma-Master-Kurs wird von Paul Grützner (Ludwigshafen), Franz Kralinger (Wien) und Marius Keel (Bern) geleitet. Ausrichter sind die 3 Sektionen der AOTrauma in Deutschland, Österreich und der Schweiz (D-A-CH). Der Kurs fokussiert sich auf komplexe Verletzungen und Verletzungsfolgen folgender anatomischer Regionen:

- Schaftfrakturen an den oberen Extremitäten (Klavikula, Humerusschaft, Unterarm)

- Frakturen am Thorax

- Frakturen und Begleitverletzungen am Handgelenk

- Schaftfrakturen an den unteren Extremitäten (Femur, Tibia)

Jedes Modul umfasst eine Reihe von Impulsreferaten mit Darstellung der aktuellen, evidenzbasierten Therapiestandards. Die Diskussion von Fällen in kleinen Gruppen dient dazu, den Teilnehmenden die Indikationsstellung und das Gesamtmanagement der Verletzung nachvollziehbar und einleuchtend aufzuzeigen. Hier hat sich in der Vergangenheit deutlich herausgestellt, dass die offene Diskussion in der eigenen Muttersprache besonders fruchtbar und inspirierend für alle Beteiligten war. Geschätzt wurde auch der intensive Austausch mit den Kollegen und Kolleginnen aus Österreich und der Schweiz. Dieses Jahr neu: Alle Teilnehmenden

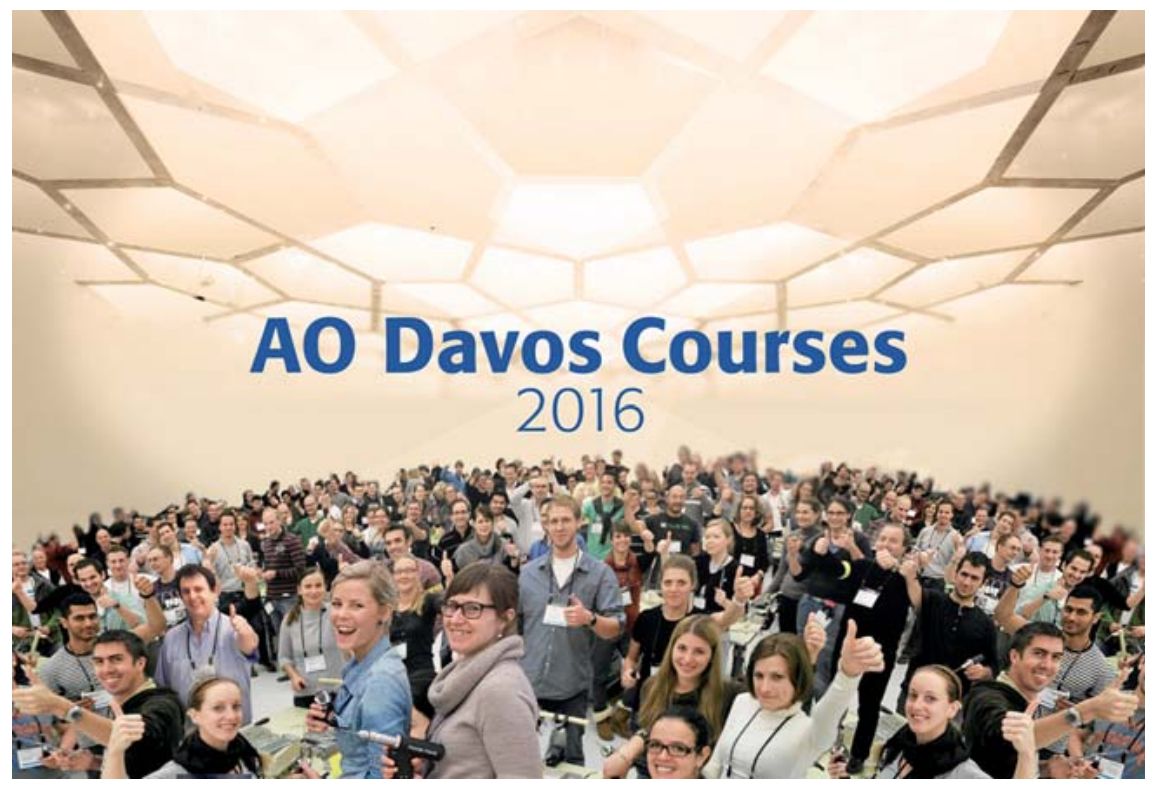

Abb. 1 AOTrauma-Kurse in Davos. Jürgen Staiger, mit freundlicher Genehmigung der AO Foundation.

werden zur Vorstellung eines eigenen Falles motiviert.

Abgesehen vom internationalen Flair während der Davoser Trauma-Kurswoche mit über 1000 Teilnehmenden und Faculty aus aller Welt sind die Selfdirected Learning Modules nur in Davos während der AO-Kurswoche möglich.

Die Teilnehmenden sollten folgende Kriterien erfüllen:

- Teilnahme am AOTrauma Prinzipienkurs und dem Fortgeschrittenenkurs für operative Frakturbehandlung
- aktuelle Tätigkeit in der Traumaversorgung in einer spezialisierten unfallchirurgischen/orthopädischen Abteilung

- mindestens 8-10 Ausbildungsjahre (Facharzt für Chirurgie, Orthopädie oder Unfallchirurgie und Orthopädie)

Mehr Informationen und Anmeldung unter: http://www.aodavoscourses.org/ Courses/AOTrauma/Masters-German. html

\section{Prof. Dr. Paul Alfred Grützner}

Ludwigshafen 